\title{
Temperature dependence of the photoluminescence of self-assembled InAs/GaAs quantum dots in pulsed magnetic fields
}

\author{
T. Nuytten* \\ INPAC-Institute for Nanoscale Physics and Chemistry, Pulsed Fields Group, K.U. Leuven, Celestijnenlaan 200D, \\ B-3001 Leuven, Belgium \\ M. Hayne ${ }^{\dagger}$ \\ Department of Physics, Lancaster University, Lancaster LA1 4YB, United Kingdom \\ and INPAC-Institute for Nanoscale Physics and Chemistry, Pulsed Fields Group, K.U. Leuven, Celestijnenlaan 200D, \\ B-3001 Leuven, Belgium \\ M. Henini \\ School of Physics and Astronomy, University of Nottingham, Nottingham NG7 2RD, United Kingdom \\ V. V. Moshchalkov \\ INPAC-Institute for Nanoscale Physics and Chemistry, K.U. Leuven, Celestijnenlaan 200D, B-3001 Leuven, Belgium \\ (Received 15 May 2007; revised manuscript received 15 February 2008; published 25 March 2008)
}

\begin{abstract}
We have studied the magnetic field $(<50 \mathrm{~T})$ dependence of the photoluminescence (PL) of self-assembled InAs/GaAs quantum dots as a function of temperature $(T)$. As the temperature is raised from 4.2 up to $80 \mathrm{~K}$, thermal redistribution causes the PL to be increasingly dominated by dots with a lower PL energy. Magneto-PL demonstrates that these low-energy dots are larger in size only in the growth direction and not in the plane of the sample. At high temperatures $(T>100 \mathrm{~K})$, a different physical phenomenon emerges: we see an anomalous decrease of the PL shift in magnetic field, which is attributed to field enhancement of the quantum dot barrier potential. This mechanism strongly favors excitons in small dots with a weak PL shift in magnetic field, hence laterally smaller dots increasingly dominate the PL at high temperatures and high fields.
\end{abstract}

DOI: 10.1103/PhysRevB.77.115348

PACS number(s): 78.67.Hc, 73.21.La, 78.55.Cr

\section{INTRODUCTION}

Self-assembled quantum dot (QD) semiconductor nanostructures are currently attracting substantial interest due to their high potential for novel optoelectronic devices. ${ }^{1-3}$ The advantages of QD-based devices originate largely from the spatial confinement of the carriers in all three dimensions, resulting in discrete atomlike energy levels. ${ }^{4}$ For devices such as lasers, the occupancy of these energy levels is ideally temperature independent for $T \leqslant 360 \mathrm{~K}$, but such ideal behavior is not observed in practice. Hence, a thorough understanding of the effect of temperature is essential for device fabrication. Here, we show that the application of a very large magnetic field can give important insight into this problem. Although the application of a magnetic field has proven to be a very powerful tool for studying the optical and electronic properties of semiconductor materials, ${ }^{5}$ the magnetooptical study of self-assembled QDs requires the application of very strong magnetic fields, of the order of tens of tesla, in order to provide anything more than a small perturbation to the orbital motion of strongly confined carriers.

In this work, we report nonresonant photoluminescence (PL) studies of InAs/GaAs QDs as a function of temperature and magnetic field. In order to investigate some key properties that govern its electronic behavior, such as the exciton wave-function extent and effective mass, we use very high pulsed magnetic fields of up to $50 \mathrm{~T}$ to probe the PL of the QD sample in the temperature range between liquid helium temperature and $160 \mathrm{~K}$. The data are then analyzed via a simple model enabling us to extract information about the exciton wave-function. Our data clearly indicate a distinction between two different temperature regimes. At lower temperatures $(T<80 \mathrm{~K})$, the PL energy shift between 0 and $50 \mathrm{~T}, \Delta E$, increases with temperature when the magnetic field is applied perpendicular to the growth direction $z$, revealing an enhanced transfer of carriers toward QDs that are bigger in the growth direction. No such trend is observed for $B \| z$, which means that the exciton extent in the plane remains unchanged for $T<80 \mathrm{~K}$. At higher temperatures ( $T$ $>100 \mathrm{~K}$ ), our results show that the zero-field PL energy decreases with increasing temperature according to the empirical Varshni law ${ }^{6}$ for the InAs band gap, i.e., with no change in confinement. In contrast, $\Delta E$ is characterized by a strong and anomalous decrease with increasing temperature, implying an increasingly strong confinement of the carriers as the temperature is raised. We resolve these contradictory observations by proposing that the magnetic field effectively increases the QD barrier potential in a way that is particularly favorable for the higher energy dots, such that their PL is less easily quenched at high temperatures.

The paper is structured as follows. In Sec. II, we outline the sample morphology and experimental conditions under which this work has taken place, and we comment on the model that we have used to analyze the dependence of the PL on magnetic field. Section III discusses the results and Sec. IV describes their interpretation. Finally, we will summarize the conclusions in Sec. V. 


\section{EXPERIMENTAL DETAILS AND ANALYSIS METHOD}

The sample was prepared using solid-source molecular beam epitaxy. A $0.5 \mu \mathrm{m}$ thick GaAs layer was grown on top of a semi-insulating GaAs substrate. Subsequently, 1.8 monolayers of InAs were deposited at a temperature of $480{ }^{\circ} \mathrm{C}$. During this phase, QDs were formed following the Stranski-Krastanow growth mode. Finally, the sample was capped with $25.2 \mathrm{~nm}$ of GaAs. The sample was excited by the light from a solid-state laser at $532 \mathrm{~nm}$ via a $200 \mu \mathrm{m}$ core optical fiber with a power density of $1-10 \mathrm{~W} \mathrm{~cm}^{-2}$. The sample itself was located in the bore of a home-made pulsed field coil, placed in a He flow system allowing PL measurements over a broad temperature range $(4.2-160 \mathrm{~K})$. The coil was connected to a $5 \mathrm{kV}, 500 \mathrm{~kJ}$ capacitor bank, enabling magnetic field pulses of $20 \mathrm{~ms}$ and up to $50 \mathrm{~T}$. The PL signal was collected by a single $550 \mu \mathrm{m}$ core collection fiber and subsequently analyzed by a $30 \mathrm{~cm}$ monochromator and a liquid nitrogen cooled InGaAs diode array using count times of up to $4 \mathrm{~ms}$. Although this sample has already been studied in high magnetic fields at low temperatures, ${ }^{7,8}$ there are, to our knowledge, no previous reports of QDs studied as a function of temperature in such high fields.

Combining the effect of the magnetic field on the electron and hole energy levels through the use of the exciton effective mass and exciton radius, $\left\langle\rho^{2}\right\rangle^{1 / 2}$, enables us to construct a model for the PL energy as a function of magnetic field. ${ }^{9}$ The influence of a magnetic field on an exciton can be divided into a low-field and a high-field limit based on the size of the magnetic length, $l_{0}=(\hbar / e B)^{1 / 2}$, where $\hbar$ is the reduced Planck constant. In the low-field regime, the magnetic length is much larger than the size of the confined exciton wavefunction, and the magnetic field can be treated as a small perturbation to the orbital motion of the confined carriers, yielding a diamagnetic shift of the energy which is quadratic with field: ${ }^{10-12} \Delta E_{L}=\left(e^{2}\left\langle\rho^{2}\right\rangle / 8 \mu\right) B^{2}$, where $\mu$ is the reduced exciton mass. In the high-field limit, i.e., when the magnetic length is much smaller than the exciton wave-function extent, the magnetic confinement of the carriers dominates the spatial confinement, and the energy levels shift with magnetic field following the quantized Landau levels: ${ }^{13} E_{n}=(n$ $\left.+\frac{1}{2}\right) \hbar \omega_{c}\left(\omega_{c}\right.$ is the cyclotron frequency). The resulting energy shift of the PL peak energy is linear with magnetic field, and when limiting our discussion to the electron and hole ground states, we can write $\Delta E_{H}=(e \hbar / 2 \mu) B$. Although these results only strictly apply to the two limits, a practical expression describing the entire magnetic field range can readily be derived by simply demanding continuity in $E(B)$ and its derivative. The entire data set for a given temperature is then fitted with an equation describing the PL energy as a function of magnetic field as follows:

$$
E_{\mathrm{cm}}= \begin{cases}E_{0}+\frac{e^{2}\left\langle\rho^{2}\right\rangle}{8 \mu} B^{2} & \text { for } B<\frac{2 \hbar}{e\left\langle\rho^{2}\right\rangle} \\ E_{0}-\frac{\hbar^{2}}{2 \mu\left\langle\rho^{2}\right\rangle}+\frac{e \hbar}{2 \mu} B & \text { for } B>\frac{2 \hbar}{e\left\langle\rho^{2}\right\rangle},\end{cases}
$$

where $E_{\mathrm{cm}}$ is the center of mass PL energy and $E_{0}$ is the zero field PL energy. Through the combination of the parabolic and linear behavior of the PL shift into one equation, where

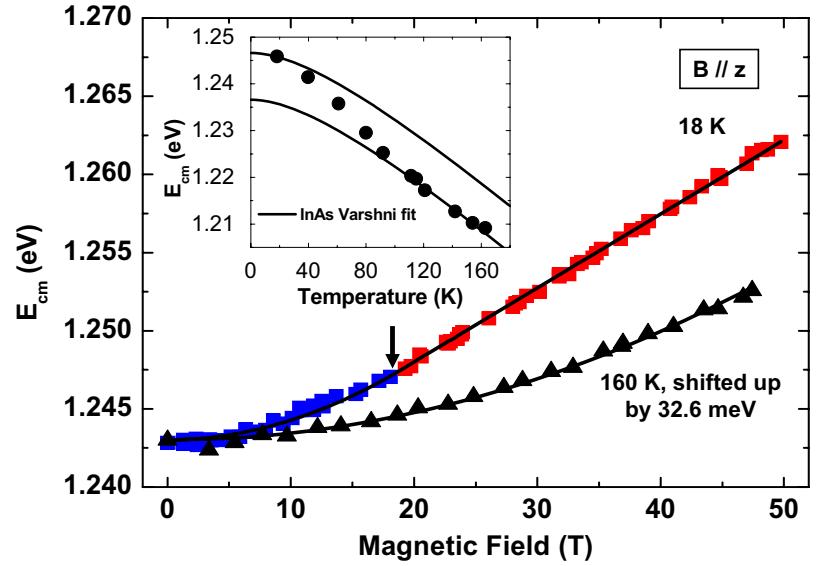

FIG. 1. (Color online) The PL energy, $E_{\mathrm{cm}}$, versus magnetic field. The data for $160 \mathrm{~K}$ have been shifted for clarity. The lines are fits to the data using Eq. (1). At lower temperatures, a crossover from parabolic to linear behavior occurs at $19 \mathrm{~T}$ (indicated by the arrow), whereas the $160 \mathrm{~K}$ data are parabolic up to $50 \mathrm{~T}$. Note that the energy shift is much larger for low temperatures. The inset shows the zero-field PL energy as a function of temperature. For low temperatures, the decrease of the PL energy with temperature is faster than predicted by the Varshni law (Ref. 6) for the InAs band gap (full lines).

the crossover field is given by $B_{c}=2 \hbar / e\left\langle\rho^{2}\right\rangle$, we are able to extract both the effective exciton radius $\left\langle\rho^{2}\right\rangle^{1 / 2}$ and the reduced exciton mass $\mu$. It should be noted that our experimental methods and analysis essentially probe the exciton radius. However, the relation between the exciton size due to quantum confinement and the physical size of the QDs ${ }^{12}$ enables us to formulate conclusions about the average size of PLactive QDs at a certain temperature. For the purposes of this investigation, we will concentrate on the shift in energy of the PL peak between 0 and $50 \mathrm{~T}, \Delta E$, and the exciton radius, but not the exciton mass.

Upon application of a magnetic field, the structure of the exciton is affected mainly through a compression of the wave function in the plane perpendicular to the applied field. Therefore, when examining the PL shift with $B \|_{z}$, the exciton radius in the plane of the sample is measured. On the other hand, with $B \perp z$, we probe the vertical and one lateral dimension of the dots. However, since it is the direction with the strongest confinement (smallest exciton radius) that determines both the size of the shift with field and the crossover field $B_{c},{ }^{14}$ and since the dots are smaller in the growth direction than in the plane of the sample, applying a magnetic field in the plane of the sample probes the vertical extent of the exciton. Indeed, we find that the shift and effective exciton radius are smaller for this field orientation, in agreement with earlier magneto-PL measurements on selfassembled QD nanostructures. ${ }^{7,8,12,15}$

\section{RESULTS}

The inset of Fig. 1 shows the zero-field PL energy at different temperatures. For temperatures below $80 \mathrm{~K}$, the decrease in PL energy with temperature is faster than predicted ${ }^{6}$ 

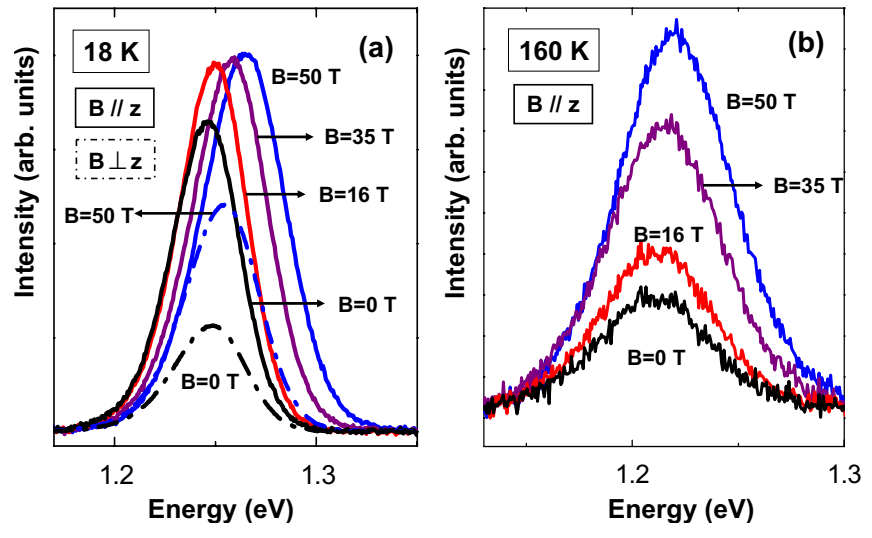

FIG. 2. (Color online) PL spectra at different magnetic fields for the $B \| z$ configuration for (a) 18 and (b) $160 \mathrm{~K}$. Two PL spectra for the $B \perp z$ orientation at low temperature are also shown in left panel (a). The PL intensity is enhanced when applying a magnetic field, in particular, for the experiments at higher temperatures. The low temperature data have a larger field shift compared to the high temperature data. Additionally, it can be seen that the PL shift with field is larger for the $B \| z$ orientation than for the $B \perp z$ configuration.

for the InAs band gap. This behavior was previously observed and attributed to thermal redistribution of electrons from higher to lower-energy dots via the wetting layer..$^{16-18}$ The increase of temperature thus not only activates the expansion of the lattice and phonon-related effects but also introduces an additional transfer of carriers between the dots, which favors lower-energy recombination. At higher temperatures, the PL energy nicely follows the changes according to the Varshni law for the InAs band gap. Following Brusaferri et al. ${ }^{16}$ who reported evidence of families of dots with "remarkably uniform sizes," we interpret this temperature dependence of the PL energy as suggesting the presence of different dot families with distinct sizes in our sample.

In order to gain further insight into the confinement of the carriers inside the dots, we then studied the magneto-PL of the sample at different temperatures and with different orientations of the field with respect to the growth direction. Figure 2 shows PL spectra for different strengths of the magnetic field for 18 and $160 \mathrm{~K}$. From these spectra, it is already clear that the PL energy shift with $B$ is larger at low temperatures and that the intensity of the PL signal is significantly reduced when increasing the temperature. The dependence of the PL peak energy on magnetic field is summarized in Fig. 1 for the same two temperatures. The $18 \mathrm{~K}$ data are typical of the low temperature regime, which is characterized by a crossover from parabolic to linear field dependence at a certain field $B_{c}$, indicated by the arrow. As the temperature increases above $100 \mathrm{~K}$, the shift of the PL energy is reduced, and there is a decreasingly smaller linear regime at fields below $50 \mathrm{~T}$, indicating stronger confinement. Figure 3 shows $\Delta E$ as a function of temperature for both field orientations. From this figure, it is evident that, for lower temperatures (i.e., below $\sim 80 \mathrm{~K}$ ), as the temperature is raised, there is a strong increase in $\Delta E$ with the magnetic field perpendicular to the growth direction $(B \perp z)$, whereas an increase in temperature hardly affects the energy shift for $B \| z$. In contrast,

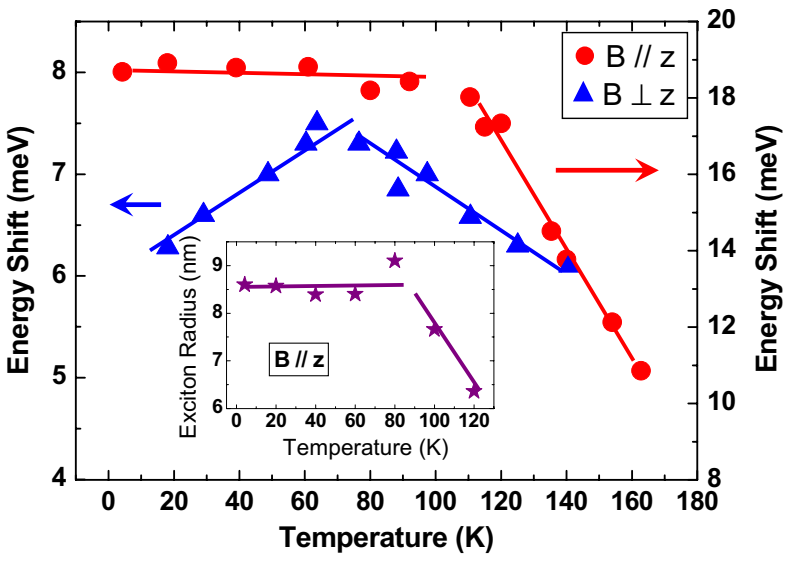

FIG. 3. (Color online) Energy shift between 0 and $50 \mathrm{~T}$ versus temperature for a magnetic field applied parallel (circles) and perpendicular (triangles) to the growth direction. A strong decrease of the PL shift characterizes the high temperature $(T>100 \mathrm{~K})$ regime. The inset shows the exciton wave-function extent as a function of temperature following Eq. (1) for $B \| z$ and reveals an apparent wave-function shrinkage at high temperatures. For $T>120 \mathrm{~K}$, the lateral exciton radius becomes so small that the crossover to the high-field regime is at too high a field to be determined reliably. The lines are guides for the eyes.

for $T>100 \mathrm{~K}$, there is a drastic reduction in the size of the shift for both field orientations. Given such different behavior, it seems clear that different mechanisms are at work in the two temperature regimes of our experiment. We will therefore go on to discuss each of these separately.

\section{DISCUSSION}

\section{A. $T<80 \mathrm{~K}$}

For the low temperature regime, the dependence of the shift $\Delta E$ on temperature is substantially different for the two different field orientations, as can be seen in Fig. 3. While the shift is more or less constant with a magnetic field applied in the growth direction, experiments with $B \perp z$ reveal an increase of the shift with temperature. As mentioned above, when $B \perp z$, we are probing the vertical extent of the exciton, since this is the direction with the strongest confinement with this field orientation. Because the exciton wavefunction is so small in this direction, $\Delta E$ is dominated by the low-field regime where it is proportional to $\left\langle\rho^{2}\right\rangle / \mu$. An increase in $\Delta E$ is thus either because $\mu$ is decreasing or $\left\langle\rho^{2}\right\rangle^{1 / 2}$ is increasing (or both). However, if it was only because $\mu$ was decreasing, this would also increase the confinement energy, and result in an increase of the zero-field PL energy relative to the change expected from the Varshni law, which is the opposite of what we observe. On the other hand, a decrease in the zero-field PL energy is entirely consistent with an enhanced transfer of carriers toward larger QDs leading to a decrease in confinement. Hence, the increase in $\Delta E$ with the magnetic field perpendicular to the growth direction indicates an expansion of the exciton wave-function in the growth direction reflecting the increased contribution of 
larger QDs to the PL emission. In contrast, the energy shift with magnetic field parallel to the growth direction remains unaltered at temperatures below $80 \mathrm{~K}$. Thus, the explanation of the data in the inset of Fig. 1 that there is thermal excitation from smaller to bigger dots is indeed supported by our magneto-PL measurements, but the magneto-PL data crucially reveal that these dots are larger in size only in the growth direction: there is no indication of a change in the average lateral size of the ensemble of dots in this temperature range.

\section{B. $T>100 \mathrm{~K}$}

We now turn to the high temperature regime. Here, the field-induced PL shift is characterized by a massive decrease as a function of temperature in both field orientations (Fig. 3 ). Applying an analysis to the data following the magnetic field dependence of the peak energy as described in Eq. (1) would lead us to interpret this as a shrinkage of the exciton radius (or, equivalently, an increase in confinement) as a result of increasing temperature (inset of Fig. 3). However, if the QDs active at higher temperatures were, on average, smaller than the ones responsible for the PL at low temperatures, their confinement energy should be higher, as would be the corresponding transition energies within the QD. In this case, the zero-field PL energy would not follow the empirical Varshni law for the InAs band gap but show a weaker temperature dependence or even an increase as a function of temperature, i.e., the exact opposite of what we observe at low $T$. However, this is clearly not the case, and we therefore conclude that the decrease of the field-induced PL shift with temperature is not due to a decrease of the (zero-field) exciton radius as a function of temperature, but that it has some other cause.

The contradiction is resolved when noting that the energy shift is determined in the presence of a very strong magnetic field, which, we propose, affects the confinement potentials. In general, applying a magnetic field causes the energy levels of bulk GaAs (the barrier material) to be lifted by $\frac{1}{2} \hbar \omega_{c}$, or about $0.9 \mathrm{meV} \mathrm{T}^{-1}{ }^{19}$ In contrast, the PL energy shift between 0 and $50 \mathrm{~T}$ of the InAs QDs with $B \| z$ for the considered temperature range varies between 10 and $20 \mathrm{meV}$ (see Fig. 3), resulting in an energy shift of about $0.2-0.4 \mathrm{meV} \mathrm{T}^{-1}$. Figure 4 shows a schematic representation of the electron energy levels of an InAs QD in the GaAs matrix and illustrates the fact that the application of a strong magnetic field increases the energy gap between the states in the QDs and the states of the surrounding bulk GaAs material. When applying a magnetic field, the energy levels of the GaAs barrier material are substantially raised, while the increase of the QD levels is rather moderate. Consequently, a strong magnetic field enhances the temperature stability of the system by counteracting the escape of carriers from the dots into the surrounding barrier material. This phenomenon has previously been observed in quantum well laser structures in an attempt to simulate a reduction in dimensionality by placing the structure in a magnetic field. ${ }^{20,21}$ However, the influence of a magnetic field on the energy levels of the QDs is strongly dependent on the degree of confinement present

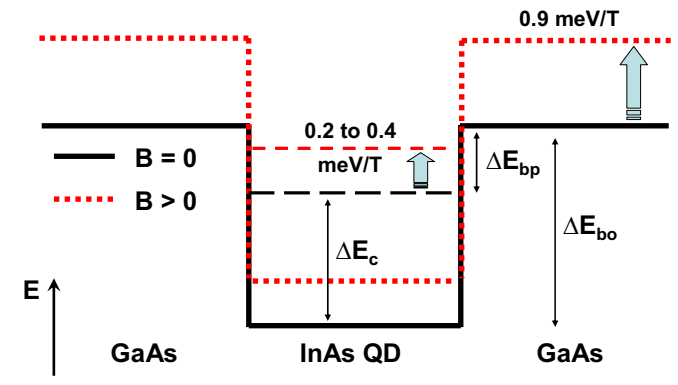

FIG. 4. (Color online) Schematic representation of the electron energy levels of the InAs QDs and GaAs matrix material as they are lifted by the magnetic field. The differential rise in energy levels as a function of $B$ increases the effective barrier height by $0.5-0.7 \mathrm{meV} \mathrm{T}^{-1}$. The confinement energy $\Delta E_{c}$, the barrier potential $\Delta E_{b p}$, and the band offset $\Delta E_{b o}$ at $B=0$ are indicated.

in the dots. Smaller dots have a stronger confinement potential, resulting in a weaker dependence on magnetic field. Therefore, the energy levels of the excitons with strong lateral confinement, i.e., those with a weak energy shift in magnetic field $\left(\sim 0.2 \mathrm{meV} \mathrm{T}^{-1}\right)$, are lifted much less than the ones with a weak lateral confinement, as the latter have a larger PL shift in field $\left(\sim 0.4 \mathrm{meV} \mathrm{T}^{-1}\right)$. In both cases, however, the barrier energy is raised by the same larger amount. This results in a field-induced enhancement of $0.7-0.5 \mathrm{meV} \mathrm{T}^{-1}$ of the QD barrier potential. Correspondingly, the extra energy difference between the QD levels and the GaAs barrier material at $50 \mathrm{~T}$ is $35 \mathrm{meV}(400 \mathrm{~K})$ for the high-energy dots (i.e., the ones with strong lateral confinement), while it is only $25 \mathrm{meV}(290 \mathrm{~K})$ for the low-energy dots (i.e., with weaker lateral confinement). In other words, the effect of the magnetic field is to deepen the confinement potentials of the QDs, and counterintuitively, the mechanism is much stronger for smaller, high-energy dots, as the energy levels of these dots are the least affected by a magnetic field. The result is that at high temperatures and high magnetic fields, the contribution of the high-energy dots to the PL relative to the low-energy dots is increased, which, in turn, results in a smaller PL shift in magnetic field. This explanation is supported by the observation that at high temperatures, the PL intensity is strongly increased upon applying a magnetic field, as can be seen in Fig. 2(b). In contrast, at low temperatures, when there is insufficient thermal energy to excite the carriers entirely out of the InAs dots and wetting layer into the barrier material, the magnetic field has very little impact on the PL intensity [Fig. 2(a)]. This corroborates the assertion that the excitation between large and small dots at low temperature occurs via the wetting layer. ${ }^{16-18}$

\section{CONCLUSIONS}

We have studied exciton confinement in self-assembled InAs/GaAs QDs. At low temperatures $(T<80 \mathrm{~K})$, we find that the PL energy decreases faster with temperature than predicted by the Varshni law for the InAs band gap. This is 
attributed to thermal excitation via the wetting layer to dots which are larger only in the growth direction of the sample. At higher temperatures $(T>100 \mathrm{~K})$, we observe a strong decrease of the PL shift in field, which we attribute to field enhancement of the QD barrier potential. As the influence of a magnetic field on the energy levels of the QDs depends inversely on the confinement energy, this mechanism is particularly important for smaller dots.

\section{ACKNOWLEDGMENTS}

This work was supported by the Belgian IAP, the SANDiE Network of Excellence (NMP4-CT-2004-500101), the Engineering and Physical Sciences Research Council (United Kingdom), a Royal Society International Joint Project, and the EuroMagNET project (Contract No. RII3CT-2004-506239) of the European Commission. M. Hayne acknowledges support of the Research Councils, UK.

\footnotetext{
*thomas.nuytten@fys.kuleuven.be

†m.hayne@lancaster.ac.uk

${ }^{1}$ D. J. Mowbray and M. S. Skolnick, J. Phys. D 38, 2059 (2005).

${ }^{2}$ N. N. Ledentsov, V. A. Shchukin, T. Kettler, K. Posilovic, D. Bimberg, L. Ya. Karachinsky, A. Yu. Gladyshev, M. V. Maximov, I. I. Novikov, Yu. M. Shernyakov, A. E. Zhukov, V. M. Ustinov, and A. R. Kovsh, J. Cryst. Growth 301-302, 914 (2007).

${ }^{3}$ D. Bimberg, M. Grundmann, and N. N. Ledentsov, Quantum Dot Heterostructures (Wiley, London, 1999).

${ }^{4}$ D. Gammon, Nature (London) 405, 899 (2000).

${ }^{5}$ A. Polimeni, G. Baldassarri Höger von Högersthal, F. Masia, A. Frova, M. Capizzi, S. Sanna, V. Fiorentini, P. J. Klar, and W. Stolz, Phys. Rev. B 69, 041201(R) (2004).

${ }^{6}$ Y. P. Varshni, Physica (Amsterdam) 34, 149 (1967).

${ }^{7}$ J. Maes, M. Hayne, V. V. Moshchalkov, A. Patanè, M. Henini, L. Eaves, and P. C. Main, Appl. Phys. Lett. 81, 1480 (2002).

${ }^{8}$ S. Godefroo, J. Maes, M. Hayne, V. V. Moshchalkov, M. Henini, F. Pulizzi, A. Patanè, and L. Eaves, J. Appl. Phys. 96, 2535 (2004).

${ }^{9}$ M. Hayne, J. Maes, S. Bersier, M. Henini, L. Müller-Kirsch, Robert Heitz, D. Bimberg, and V. V. Moshchalkov, Physica B 346-347, 421 (2004).

${ }^{10}$ T. Someya, H. Akiyama, and H. Sakaki, Phys. Rev. Lett. 74, 3664 (1995).
}

${ }^{11}$ S. N. Walck and T. L. Reinecke, Phys. Rev. B 57, 9088 (1998).

${ }^{12}$ M. Sugisaki, H.-W. Ren, S. V. Nair, K. Nishi, and Y. Masumoto, Phys. Rev. B 66, 235309 (2002).

${ }^{13}$ S. Nomura, L. Samuelson, M.-E. Pistol, K. Uchida, N. Miura, T. Sugano, and Y. Aoyagi, Appl. Phys. Lett. 71, 2316 (1997).

${ }^{14}$ J. Maes, M. Hayne, M. Henini, F. Pulizzi, A. Patanè, L. Eaves, and V. V. Moshchalkov, Physica B 346-347, 428 (2004).

${ }^{15}$ Y. Sidor, B. Partoens, F. M. Peeters, N. Schildermans, M. Hayne, V. V. Moshchalkov, A. Rastelli, and O. G. Schmidt, Phys. Rev. B 73, 155334 (2006).

${ }^{16}$ L. Brusaferri, S. Sanguinetti, E. Grilli, M. Guzzi, A. Bignazzi, F. Bogani, L. Carraresi, M. Colocci, A. Bosacchi, P. Frigeri, and S. Franchi, Appl. Phys. Lett. 69, 3354 (1996).

${ }^{17}$ A. Polimeni, A. Patanè, M. Henini, L. Eaves, and P. C. Main, Phys. Rev. B 59, 5064 (1999).

${ }^{18}$ P. Dawson, E. O. Göbel, K. Pierz, O. Rubel, S. D. Baranovskii, and P. Thomas, Phys. Status Solidi B 244, 2803 (2007).

${ }^{19}$ Here, we have calculated the increase in barrier height for the electrons, since we are discussing thermal excitation of the carriers (electrons) out of the dot. However, the total increase in energy for the dots would be the increase for the excitons (holes+electrons), which makes our assessment an underestimate of the effect.

${ }^{20}$ Y. Arakawa and H. Sakaki, Appl. Phys. Lett. 40, 939 (1982).

${ }^{21}$ T. T. J. M. Berendschot, H. A. J. M. Reinen, H. J. A. Bluyssen, C. Harder, and H. P. Meier, Appl. Phys. Lett. 54, 1827 (1989). 